\title{
Separation of composite spectra: the spectroscopic detection of an eclipsing binary star ${ }^{\star}$
}

\author{
J. F. González and H. Levato
}

\author{
Complejo Astronómico El Leoncito (CASLEO), San Juan, Argentina \\ e-mail: fgonzalez@casleo.gov.ar
}

Received 1 April 2005 / Accepted 5 October 2005

\section{ABSTRACT}

We present an iterative method that allows us to compute the individual spectra and radial velocities of the two stellar components of spectroscopic binaries. We applied this method to HD 143511 and detected the occurrence of eclipses at the conjunction phases. The orbital analysis provides minimum masses with errors of $1 \%$. Absolute masses and radii were derived in two different ways: a) by combining information provided by radial velocities, rotational velocities, and spectral line intensities; and b) using light curves from the ASAS-3 database.

Key words. techniques: radial velocities - stars: binaries: spectroscopic - stars: fundamental parameters - stars: individual: HD 143511 Galaxy: open clusters and associations: individual: NGC 6025

\section{Introduction}

In the course of our program for detecting spectroscopic binaries among members of open clusters, we often encountered spectra with both components close enough to make proper measurement of the individual radial velocities (RVs) very difficult. Historically, the problem of measuring reliable RVs and analyzing the component spectra in double-lined spectroscopic binaries is a tough one to solve. Fortunately, in the past few years several techniques have been developed to overcome these problems. On the one hand, the two-dimensional cross-correlations method by Zucker \& Mazeh (1994) has been shown to be an excellent tool for measuring precise RVs of double-lined spectroscopic binaries when the spectral morphology of the components is known. On the other hand, the tomographic method by Bagnuolo \& Gies (1991), the spectral disentangling by Simon \& Sturm (1994), and the Fourier disentangling by Hadrava (1995) are all primarily aimed at the complementary problem: calculation of the spectra of the stellar components from a sample of binary spectra at different orbital phases with known RVs. In the case of both disentangling methods, the computation of the RVs, or alternatively the orbital parameters, can be done indirectly through optimization of the spectra disentangling process.

In this paper we propose a procedure for dealing with both problems: separating the spectral components and measuring

* The observations presented here were made at the Complejo Astronómico El Leoncito (CASLEO), which is operated under an agreement between the Consejo Nacional de Investigaciones Científicas y Técnicas de la República Argentina (CONICET) and the National Universities of La Plata, Córdoba, and San Juan. their RVs. This is a versatile method that can be easily applied using standard tools for spectroscopic data analysis, such as those available in the IRAF or MIDAS packages. This method was used originally by Marchenko et al. (1998) to measure the companion of a Wolf-Rayet binary. However, afterwards it was largely ignored, and it has not used nor mentioned since as a general method for spectral disentangling of binary stars (Gies 2004; Holmgren 2004; Hadrava 2004). In Sect. 2 of this paper we describe how we implemented the method and, as an illustrative example, in Sect. 3 we apply it to analysis of the binary HD 143511. In the subsequent sections we report the detection of eclipses from our spectroscopic observations and derive preliminary absolute masses and dimensions of the system.

Located only $12^{\prime}$ from the center of the open cluster NGC 6025, HD 143511 was detected as a spectroscopic binary as a result of our spectroscopic program for searching and characterizing binary stars in clusters. It has been classified as A0IV-V in the Michigan Catalogue (Houk \& Cowley 1975), but no other spectroscopic study has been published. It was also included in the catalogue of suspected variable stars (identified as NSV 7400, Kukarkin et al. 1981) after Strohmeier (1967) reported a photometric variation of 0.25 mag. Very recently, Otero \& Dubovsky (2004) have reported HD 143511 as an eclipsing binary with a period of 5.5354 days.

\section{The method}

The proposed method is an iterative procedure that allows computation of the spectra and the RVs of the two stellar components in a spectroscopic binary system. The basic idea is to use alternately the spectrum of one component to calculate the 
spectrum of the other one. In each step, the calculated spectrum of one star is used to remove its spectral features from the observed spectra and then the resulting single-lined spectra are used to measure the RV of the remaining component and to compute its spectrum by combining them appropriately.

The calculations are carried out in two blocks. The first one calculates the spectra of the primary and secondary components, and the second one measures the RVs. The computation of the two blocks depends on the results of each other, so they are run iteratively up to convergence.

\subsection{Computation of the individual spectrum of each component}

The individual spectra of the stellar components are computed by Doppler shifting and combining the observed spectra in an iterative process. The procedure for the computation of the $j$ th iteration is as follows. Let $S_{i}$ be the $i$ th observed spectrum, with $i=1, n$, and $A^{j}(x)$ and $B^{j}(x)$ the computed spectra of the primary and the secondary components, respectively, in the $j$ th approach. All the spectra are calibrated as a function of $x \equiv \ln \lambda$. With this variable choice that is usual for RV works, a Doppler shift results in a uniform shift, while the spectral morphology is preserved.

The first step is to shift the spectrum $B^{j}$ to match the lines of the secondary component in the $i$ th spectrum. Then we compute the differences $S_{i}(x)-B^{j}\left(x-v_{b, i}\right)$, which correspond to the $i$ th observed spectrum but now contain only spectral features of the primary component. In that manner, the computation of the primary spectrum $A$ is performed as

$A^{j}=\left\langle S_{i}\left(x+v_{a, i}\right)-B^{j-1}\left(x-v_{b, i}+v_{a, i}\right)\right\rangle_{i} ;$

and similarly for the $B$ spectrum,

$B^{j}=\left\langle S_{i}\left(x+v_{b, i}\right)-A^{j}\left(x-v_{a, i}+v_{b, i}\right)\right\rangle_{i}$,

where \langle\rangle$_{i}$ denotes average over $i=1, n$ and $v_{a}$ and $v_{b}$ are the velocities of the components in units of the speed of light: $v_{a}=$ $\mathrm{RV}_{A} \cdot c^{-1}$.

In each iteration, the residuals of the secondary lines still present in the $A$ spectrum are reduced approximately by a factor $1 / n$, where $\mathrm{n}$ is the number of observed spectra supposed to be at different phases. Thus, the algorithm converges in a few iterations. The convergence depends on the number of spectra and the number of spectral lines in the spectrum, but rarely more than 5-7 iterations are needed.

Even though the spectrum of one component is used to compute the spectrum of the other one, in practice, it is not necessary to have $A$ and $B$ beforehand to start the calculations. In most cases, adopting a flat spectrum for the secondary component (the weakest) works well as a starting point for the iterations. Note that the starting primary spectrum $A^{0}$ is not needed at all, since $A^{1}$ is computed from $B^{0}$. Thus, getting the initial spectra of the individual components by no means represents a problem in itself. In the case of a sample composed of only 2 or 3 observed spectra, the convergence is slower and, if the calculations start from a flat spectrum for $B^{0}$, we suggest a modification in the algorithm to improve the convergence.
The spectrum $B^{1}(x)$ involved in the calculation of $A^{2}(x)$ should be replaced by $n /(n-1) \cdot B^{1}(x)$, which improves the calculation of the primary spectrum in the second iteration, as can be proved mathematically.

For the first computation of the separate spectra, an estimated value of the RV of both components is required. In this paper we have applied classical cross-correlations for this purpose. Our implementation of the method was entirely carried out using IRAF tasks, combined into an IRAF script. We employed the task fxcor for measuring the RVs, sarith for spectrum arithmetic, dopcor for shifting the spectra, and scombine for computing the spectrum averages.

We note that the averages involved in the computation of the spectra can be performed using any combination algorithm. For example, the use of the IRAF task scombine allows the inclusion of weights or of some rejection algorithm, preventing the replication of bad pixels or cosmic rays.

\subsection{Radial velocities}

After obtaining the spectra of the binary components $A$ and $B$, we can compute the spectra $S_{i}(x)-B\left(x-v_{b, i}\right)$ and $S_{i}(x)-A\left(x-v_{a, i}\right)$, i.e., the observed spectra after the removal of one of the components. These spectra are then used to measure the RVs of the primary and secondary components, respectively. For this purpose, any standard RV technique can be applied, since these are single-lined spectra. As mentioned above, we used classical cross-correlation by means of the IRAF task fxcor. At this point, it must be emphasized that any template choice, spectral sample selection, or Fourier filtering can be done in order to improve the RV measurement. The zero of $\mathrm{RV}$ measurements is fixed by the $\mathrm{RV}$ of the template. Furthermore, the spectral shifts involved in the algorithms of $\mathrm{RV}$ measurement or spectrum reconstruction equal the actual $\mathrm{RV}$ values and do not need to be a multiple of the pixel size. In particular, we used the IRAF task dopcor to perform the redshifts. Obviously, the price to be paid is repeated resampling of the spectra.

The RV computations must be carried out iteratively. In fact, the RV of the secondary component is involved in calculating the spectrum $S_{i}(x)-B\left(x-v_{b, i}\right)$ used to measure the RV of the primary component. The number of iterations needed for converging depends strongly on the initial values of the RVs. However, for a given pair of spectra $A$ and $B$, the RV calculation of one observed spectrum does not depend on the RVs of the remaining. Thus, different numbers of iterations can be used for the various spectra. Furthermore, we can measure the RV of an observed spectrum, even if it was not included in the computation of $A$ and $B$. When some spectra are strongly blended or very noisy, it may be advisable to keep them apart and use the best spectra for computing the individual spectrum of each component (cf. Sect. 3.2).

\subsection{Application and limitations}

In order to evaluate the limitations of the reconstruction method, particularly regarding the phase coverage of the 


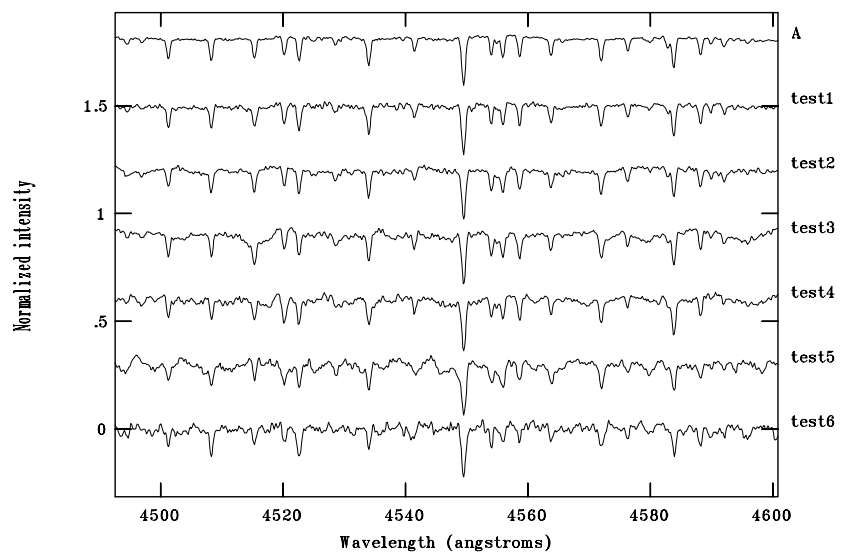

Fig. 1. Examples of the reconstruction of the primary spectrum with a small number of spectra. The first is spectrum $A$ calculated with all our observations. The following are calculated with different subsamples covering various $\mathrm{RV}$ ranges $\Delta=\max \left(v_{b}-v_{a}\right)-\min \left(v_{b}-v_{a}\right)$ : test $1=$ 5 spectra distributed along the whole orbital cycle, $\Delta=398 \mathrm{~km} \mathrm{~s}^{-1}$; test $2=8$ spectra within one fourth of the orbit (phases $=0.49-0.64$, $\Delta=118 \mathrm{~km} \mathrm{~s}^{-1}$ ); test $3=5$ spectra near a quadrature (phases = $\left.0.55-0.84, \Delta=64 \mathrm{~km} \mathrm{~s}^{-1}\right)$; test $4=5$ spectra near a quadrature (phases $=0.64-0.84, \Delta=40 \mathrm{~km} \mathrm{~s}^{-1}$ ); test $5=5$ spectra near the conjunctions $\left(\Delta=35 \mathrm{~km} \mathrm{~s}^{-1}\right)$; test $6=2$ spectra at phases 0.21 and 0.56 .

observations and application to blended spectra, we carried out several tests involving observed and synthetic spectra.

Important question are what the minimum number of observed spectra is for the method to work properly or how the observations should be distributed along the orbital cycle. Although it should be possible to apply the method using only two observed spectra, the advantages of the method are more evident when the observations are taken at many different phases. In a first series of experiments, we reconstructed the spectra $A$ and $B$ of the components of the binary HD 143511 using only five of our observed spectra as described in Sect. 3. We defined various subsamples with different phase coverage. Figure 1 shows a few of the reconstructed spectra illustrating the results. Analysis of the residuals of these calculations with respect to the spectra $A$ and $B$ obtained using the whole of our spectra shows that the quality of the results depends on the RV range covered by the observations rather than on the orbital phase range. In addition, the larger the RV difference of the components in the various composite spectra, the lower the main frequencies of the modulations in the residuals.

To understand how the quality of the results of computed spectra in the general case is related to the RV distribution of the observed spectra, let us consider the reconstruction procedure in the absence of observational noise. Let $A$ and $B$ be the spectra of the two components to be calculated and $A^{j}, B^{j}$ be those in the $j$ th approach. Thus, the calculated primary spectrum differs from the real one by $\Delta A^{j}(x)=A^{j}-A$. It can be proved that in each iteration these residuals are spread out over the spectrum according to the RV distribution of the observed spectra: $\Delta A^{j+1}=\left\langle\Delta A^{j}\left(x-d_{i}+d_{k}\right)\right\rangle_{i, k}$, where $d_{i}=v_{b, i}-v_{a, i}$ is the relative velocity of the components in the $i$ th observed spectrum. In other words, we can consider that in each iteration the residuals $\Delta A^{j}$ are convolved with a symmetric function $f(x)=n^{-2} \sum_{i, k} \delta\left(x-d_{i}+d_{k}\right)$. If these $\delta$ Dirac functions completely cover a given range in relative velocities, i.e. if the separation between two adjacent $\delta$-functions is smaller than or the same order as the width of the spectral lines, then the practical effect is that, after $m$ iterations, the residuals have been convolved with a Gaussian of $\sigma=\sqrt{2 m} \cdot \sigma_{d}$, where $\sigma_{d}$ is the standard deviation of the observed RV differences $d_{i}$. Alternatively, if the RVs are uniformly distributed in a range $\Delta d$, then $\sigma=$ $\Delta d \sqrt{m / 6}$, approximately.

In this manner, the quality of the reconstructed spectra depends on the RV range independently of the corresponding orbital phase range. Thus, the results obtained from observations taken only close to the conjunction are expected to be similar to those of a lower amplitude binary.

The different spacial frequencies in the spectrum are not recovered with the same precision; low frequency modulations are more poorly reconstructed. For that reason broad features (HI lines wings, continuum shape, etc.) cannot be precisely measured during the subsequent spectral analysis of the components. In practice, features that are wider than a few times the RV amplitude are barely recovered. These low frequency modulations remain equal to those of the component spectra used for starting the iterations.

Note that, since the residuals are spread out by convolving them repeatedly with the same function, the reconstruction works faster in the first iterations. Furthermore, a large number of iterations could produce a higher resampling noise. On the other hand, RV measurements, which are based on the sharper spectral features, are usually well-measured even if the low frequencies are not properly reconstructed. Note, moreover, that filters in the Fourier space can be applied during the crosscorrelations used for the RV measurement.

In the case in which the observations do not completely cover an RV range but are taken at only a few different phases, e.g. when only the quadratures are observed, then the reconstructed spectra might present artificial peaks, simulating absorption, or emission spectral lines, evenly distributed throughout a region in the spectrum.

The most serious problem for the RV calculation arises when the amplitude of the RV curve is comparable to the width of spectral lines. In this case, the Fourier frequencies corresponding to the spectral lines are close to those frequencies that are difficult to recover. In a second series of experiments, we generated synthetic spectra with higher rotational velocity in order to evaluate the quality of the results in cases where the amplitude of the RV curves is smaller compared to the FWHM of the spectral lines. We found that good convergence of RVs is obtained, provided that the maximum RV difference between the components is at least the same order as the FWHM of the spectral lines.

\section{Radial velocities and orbital analysis of HD 143511}

\subsection{Observations}

During several observing runs between 1998 and 2004 at CASLEO, we obtained 37 spectra of HD 143511 using the 
REOSC echelle spectrograph mounted on the $2.15 \mathrm{~m}$ telescope. The spectra cover the region $3700-5900 \AA$ with a resolution $R=13300$. Typically a signal-to-noise ratio $S / N=60-90$ at maximum was reached with exposure times of $8 \mathrm{~min}$. The effective signal-to-noise is about $5-10$ for the useful metallic lines, whose depths are on the order of $10 \%$ of the continuum level.

\subsection{Radial velocities and computation of the component spectra}

Preliminary RVs were obtained by cross-correlation using as the template a spectrum of HR 6041, a low-rotation star of spectral type A1 V. For the RV measurement, we selected spectral windows that contain metallic lines and exclude the broad Balmer lines, summing up $950 \AA$.

Since several observations were taken near the conjunction phases, the spectral lines of the two components appear blended. Thus, for these preliminary RVs measured by crosscorrelation, we preferred to use a template with a lower rotational velocity than our object. Even then, it was not possible to measure the six most severely blended spectra. To quantify the spectral blending, we defined the relative spectral separation of the components as the quotient of the RV difference over the quadratic sum of the $F W H M$ for the spectral lines of the components, i.e.,

$$
\left|R V_{B}-R V_{A}\right| / \sqrt{F W H M_{A}^{2}+F W H M_{B}^{2}}
$$

With this definition, the spectra that could be resolved by crosscorrelation had relative separations larger than 0.64 . Note that the peaks in the cross-correlation function are somewhat more blended than the spectral lines, because of the contribution of the width of the template lines to the correlation peak width.

These preliminary velocities, along with a flat spectrum for the secondary component, were taken as the starting solution for the spectra reconstruction. In order to improve the convergence, for the first calculation of the individual spectra $A$ and $B$, we used only the 23 best resolved spectra, those with relative separation greater than 1 . Then, we used the spectra $A$ and $B$ to calculate the RV of all our spectra. As described in Sect. 2, RVs were measured by cross-correlation of the computed singlelined spectra. As a template we used the same spectrum of HR 6041 as was used for the preliminary velocities. Thus, the zero of our RVs was defined by the RV adopted for this template $\left(-11.35 \mathrm{~km} \mathrm{~s}^{-1}\right)$, which was derived from measurement of individual spectral lines. Satisfactory results were obtained for all but two spectra, which will be discussed later and are not considered in the following.

The number of iterations needed to reach the convergence during the RV calculations depended strongly on the RV difference between the components and the accuracy of the starting values. For well-resolved spectra, 3 iterations are usually enough, while 7-8 were needed for the most heavily-blended spectra. In the case of spectra not measured by standard crosscorrelation, we performed the computations starting from different RV values and always found convergence to the same

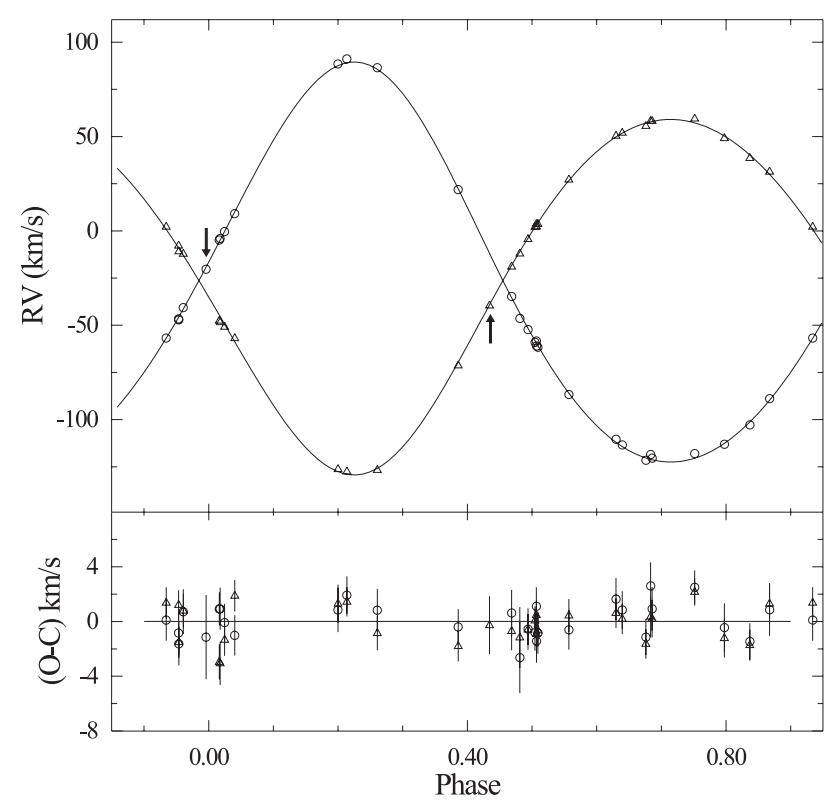

Fig. 2. RV curves of HD 143511. Triangles and circles represent the velocity of the primary and the secondary, respectively. The lower panel shows the differences observed-minus-calculated and error bars. The two points marked by arrows correspond to spectra with only one visible component.

final RVs. The algorithm proved to converge satisfactorily even if the original values are wrong by $10-20 \mathrm{~km} \mathrm{~s}^{-1}$.

It must be noted, however, that the identification of the components should be defined previously. In fact, when both components have a similar spectral type, the calculations may converge to a solution with the components swapped. At any rate, the subsequent orbital analysis can easily distinguish between the two possible solutions.

Once we got the RV of the 29 spectra, we recomputed the spectra $A$ and $B$. In that way, we alternated the computation of RVs and calculation of the spectra of the components until convergence, which took place at about the 4 th iteration. In the final calculation of the spectra $A$ and $B$ we excluded, in addition to the two single-lined spectra, three other spectra taken at phases of partial eclipse according to subsequent analysis (see Sect. 4.3).

Figure 2 shows the final RV curves, while the resulting spectra of the primary and secondary components $A$ and $B$ are shown in Fig. 3. For comparison, typical observed binary spectra are shown. Note the significant signal-to-noise improvement of $A$ and $B$, as they are averages of many observed spectra.

Table 1 shows the final RVs along with their errors and orbital phases. We adopted the RV errors provided by the IRAF task fxcor, which are calculated from the asymmetric noise of the cross-correlation function and the parameters of the correlation peak, under the assumption of purely random noise. Strictly speaking, the uncertainty of the calculated spectra $A$ and $B$, which are used to remove one of the components from the observed spectra prior to the RV measurements, should be considered as an additional error source. However, the comparison with the rms of the residuals of the fitted orbit (see Sect. 3.3) indicates that the adopted formal errors calculated in 


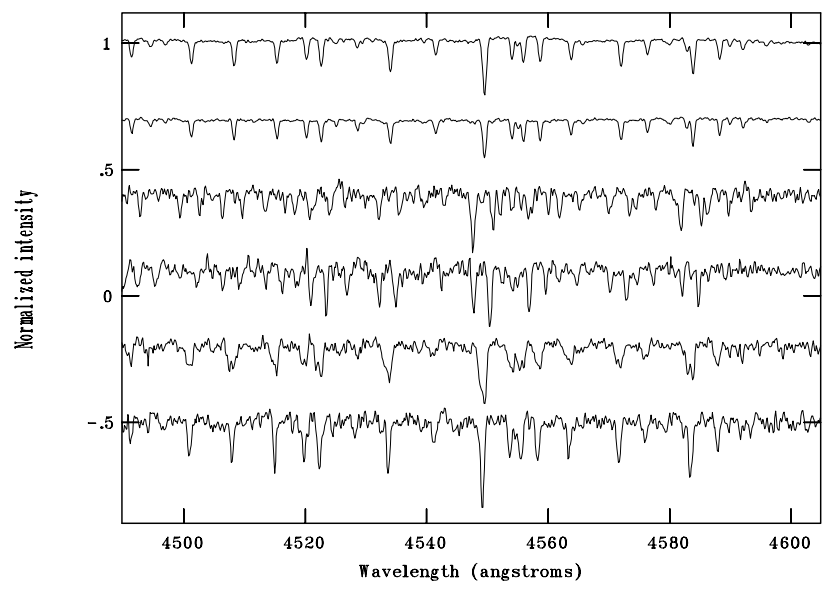

Fig. 3. Illustrative portion of the spectra of HD 143511. The first two spectra are $A$ and $B$, the computed spectra of the primary and the secondary components. The remaining spectra are typical observed spectra of the binary. The 3 th and 4 th spectra are near quadrature phases, the 5th at an intermediate phase, and the 6th is a spectrum very near to conjunction $(\phi=0.2201)$ but outside eclipse, for which reliable RV of both components were measured. All the spectra are normalized and have been shifted by steps of 0.3 for clarity.

the course of the cross-correlations are good estimates of the RV uncertainties.

Our method allows us to measure the RV of both components even for severely blended spectra, with a relative separation (defined above) of 0.28 . The two spectra at orbital phases 0.1857 and 0.7475 could not be resolved. However, the reason was not the small RV difference between the components but the eclipse of one of the components. In fact, the expected RV separation of the components in the spectrum at phase 0.1857 is $28 \mathrm{~km} \mathrm{~s}^{-1}$, significantly larger than the $\mathrm{RV}$ difference of the spectrum at phase $0.2201\left(16 \mathrm{~km} \mathrm{~s}^{-1}\right)$, which could be measured without difficulty. The two nonresolved spectra are very close to the predicted phases of eclipses: 0.1924 and 0.7517 . Furthermore, the $F W H M$ of spectral lines, as well as the RV for these two spectra, agree with the values expected for the component closer to the observer.

\subsection{Orbital parameters}

We used the least squares method to fit a Keplerian orbit to our RV data, and we obtained the following parameters: $P$ (period), $V_{\gamma}$ (center-of-mass velocity), $T_{o}$ (time of periastron passage), $K_{A}$ and $K_{B}$ (amplitudes of RV curves), $e$ (orbital eccentricity), $\omega$ (argument of periastron), and $q$ (mass ratio). Individual errors of RV measurements were used to assign weight during the orbital fitting. The resulting values of the orbital parameters along with 1-sigma errors are listed in Table 2. The RV residuals $\left(1.3-1.4 \mathrm{~km} \mathrm{~s}^{-1}\right)$ are similar to the adopted measurement errors and are typical for RV measurements of slowly-rotating single stars with our instrument.

\section{Spectral analysis}

\subsection{Spectral type}

This method provided high $\mathrm{S} / \mathrm{N}$ spectra of the individual spectra of the binary components, which allows us to perform a more detailed spectroscopic analysis of both stars. We derived spectral types by comparing the ratio of metallic lines with that of standard stars observed with the same instrument. Both stars have the same spectral type: A1 V. However, the line-ratio measurements suggest a slightly later type for the secondary (about 0.3 subtypes). We adopted stellar temperatures of $9300 \mathrm{~K}$ and $9400 \mathrm{~K}$, respectively. This is consistent with the photometric index $\mathrm{c} 1$ for this binary ( $1=1.079$, Kilambi 1975), which corresponds to $9330 \mathrm{~K}$ according to the calibration of Napiwotzki et al. (1993).

In this first analysis we found that Sr II lines appear slightly enhanced, while most of the other metals show normal intensity. In order to investigate the presence of chemical peculiarities, we plan to perform a detailed abundance analysis in the near future.

\subsection{Rotational velocity}

With the aim of estimating the stellar sizes, we tried to estimate the rotational velocities of the components from the high signal-to-noise spectra $A$ and $B$, even when the expected values of $v \sin i$ are similar to our spectral resolution. We then measured the $F W H M$ of the peak of the cross-correlation function of the spectra $A$ and $B$ against an observed spectrum of a very low rotation star (HR 8616). The FWHM was corrected for the contribution of the instrumental profile and was transformed to $v \sin i$. This transformation was calibrated by measuring lowrotation spectra convolved with several calculated rotation profiles. The resulting values are $v \sin i(A)=27 \pm 2 \mathrm{~km} \mathrm{~s}^{-1}$ and $v \sin i(B)=21 \pm 3 \mathrm{~km} \mathrm{~s}^{-1}$. The errors were estimated by applying the same procedure to a sample of eight stars included in the catalog of Royer et al. (2002) with spectral types and rotational velocities that are similar to HD 143511.

\subsection{Luminosity-ratio and the eclipsing nature of HD 143511}

The mean luminosity ratio was derived from the ratio of the equivalent width of the 20 most conspicuous metallic lines measured in the spectra $A$ and $B$. We performed a small correction to the line intensities in order to take the temperature difference between the components into account. On the other hand, the differential bolometric correction, which is required to convert the observed flux ratio into the bolometric luminosity ratio, is negligible because of the similarity of the two stars. Finally, we obtained $L(B) / L(A)=0.6 \pm 0.1$.

Considering the high probability of eclipses suggested by two of our spectra (see Sect. 3.2), we searched for light variations making use of the spectra separation algorithms. The idea is to compare each observed spectrum with the sum of the component spectra $A$ and $B$ when appropriately shifted and scaled by factors $\alpha$ and $\beta$, respectively. These factors describe the light 
Table 1. Radial velocities.

\begin{tabular}{|c|c|c|c|c|c|c|c|}
\hline $\begin{array}{c}\text { HJD } \\
-2450000\end{array}$ & Phase $^{a}$ & $\begin{array}{r}R V_{A} \\
\mathrm{~km} \mathrm{~s}^{-1}\end{array}$ & $\begin{array}{r}\epsilon_{A} \\
\mathrm{~km} \mathrm{~s}^{-1}\end{array}$ & $\begin{array}{r}R V_{B} \\
\mathrm{~km} \mathrm{~s}^{-1}\end{array}$ & $\begin{array}{r}\epsilon_{B} \\
\mathrm{~km} \mathrm{~s}^{-1}\end{array}$ & $\begin{array}{c}(\mathrm{O}-\mathrm{C})_{A} \\
\mathrm{~km} \mathrm{~s}^{-1}\end{array}$ & $\begin{array}{l}(\mathrm{O}-\mathrm{C})_{B} \\
\mathrm{~km} \mathrm{~s}^{-1}\end{array}$ \\
\hline 1586.8584 & 0.2329 & -12.1 & 2.2 & -46.4 & 2.6 & -1.2 & -2.6 \\
\hline 1589.8268 & 0.7692 & -48.5 & 1.6 & -4.0 & 1.5 & -3.1 & 0.9 \\
\hline 1591.8651 & 0.1374 & -71.4 & 1.1 & 21.9 & 1.3 & -1.8 & -0.4 \\
\hline 1592.8137 & 0.3087 & 27.1 & 1.2 & -86.7 & 1.5 & 0.4 & -0.6 \\
\hline 1954.8149 & 0.7055 & -10.9 & 1.1 & -47.1 & 1.6 & -1.5 & -1.6 \\
\hline 1957.8045 & 0.2455 & -4.4 & 1.1 & -52.3 & 1.5 & -0.6 & -0.6 \\
\hline 1957.8884 & 0.2607 & 3.6 & 1.2 & -61.7 & 1.5 & -0.7 & -0.8 \\
\hline 1958.8131 & 0.4278 & 55.6 & 1.1 & -121.5 & 1.3 & -1.6 & -1.1 \\
\hline 2061.6871 & 0.0123 & -126.8 & 1.2 & 86.5 & 1.6 & -0.9 & 0.8 \\
\hline 2068.5858 & 0.2586 & 2.2 & 1.3 & -61.0 & 1.6 & -0.9 & -1.4 \\
\hline 2444.5942 & 0.1857 & $-39.6^{b}$ & 1.3 & & & -0.3 & \\
\hline 2445.7315 & 0.3912 & 52.0 & 1.1 & -113.4 & 1.4 & 0.2 & 0.8 \\
\hline 2447.5091 & 0.7123 & -12.2 & 1.2 & -40.6 & 1.6 & 0.8 & 0.7 \\
\hline 2447.7038 & 0.7475 & & & $-20.3^{b}$ & 1.3 & & -1.1 \\
\hline 2472.6625 & 0.2564 & 2.1 & 1.0 & -59.1 & 1.3 & 0.1 & -0.8 \\
\hline 2473.6645 & 0.4374 & 58.2 & 1.4 & -120.4 & 1.6 & 0.2 & 0.9 \\
\hline 2474.5008 & 0.5885 & 38.5 & 1.1 & -102.8 & 1.3 & -1.7 & -1.5 \\
\hline 2474.6699 & 0.6190 & 31.3 & 1.5 & -88.9 & 1.9 & 1.3 & 0.9 \\
\hline 2475.6270 & 0.7919 & -56.9 & 1.1 & 9.1 & 1.5 & 1.9 & -1.0 \\
\hline 2777.8057 & 0.3815 & 50.4 & 1.1 & -110.4 & 1.5 & 0.6 & 1.6 \\
\hline 2778.7336 & 0.5491 & 49.1 & 1.4 & -113.1 & 1.8 & -1.2 & -0.4 \\
\hline 2779.5964 & 0.7050 & -7.9 & 1.1 & -46.6 & 1.6 & 1.2 & -0.8 \\
\hline 2843.5495 & 0.2583 & 3.5 & 1.1 & -58.3 & 1.4 & 0.5 & 1.1 \\
\hline 2844.5283 & 0.4351 & 58.2 & 1.5 & -118.5 & 1.7 & 0.3 & 2.6 \\
\hline 2862.5221 & 0.6858 & 2.1 & 1.1 & -56.8 & 1.5 & 1.4 & 0.1 \\
\hline 2865.4800 & 0.2201 & -19.0 & 1.4 & -34.8 & 1.7 & -0.7 & 0.6 \\
\hline 3067.7892 & 0.7680 & -47.6 & 1.3 & -4.8 & 1.2 & -3.0 & 0.9 \\
\hline 3067.8347 & 0.7762 & -50.9 & 1.2 & -0.4 & 1.4 & -1.3 & -0.1 \\
\hline 3068.8047 & 0.9514 & -126.3 & 1.4 & 88.5 & 1.6 & 1.3 & 0.8 \\
\hline 3068.8814 & 0.9653 & -127.6 & 1.1 & 91.1 & 1.4 & 1.4 & 1.9 \\
\hline 3071.8587 & 0.5031 & 59.5 & 1.0 & -118.0 & 1.2 & 2.2 & 2.5 \\
\hline
\end{tabular}

Table 2. Orbital parameters.

\begin{tabular}{lrll}
\hline \hline$P(\mathrm{~d})$ & 5.535465 & \pm & 0.000024 \\
$T_{o}{ }^{a}$ & HJD 2 452404.818 & \pm & 0.003 \\
$V_{\gamma}\left(\mathrm{km} \mathrm{s}^{-1}\right)$ & -26.35 & \pm & $0.18^{b}$ \\
$K_{A}\left(\mathrm{~km} \mathrm{~s}^{-1}\right)$ & 97.23 & \pm & 0.38 \\
$K_{B}\left(\mathrm{~km} \mathrm{~s}^{-1}\right)$ & 106.04 & \pm & 0.47 \\
$e$ & 0.0946 & \pm & 0.0023 \\
$\omega$ & 190.2 & \pm & 2.0 \\
$q$ & 0.8886 & \pm & 0.0053 \\
$a \sin i\left(R_{\odot}\right)$ & 21.90 & \pm & 0.07 \\
$M_{A} \sin ^{3} i\left(M_{\odot}\right)$ & 2.439 & \pm & 0.024 \\
$M_{B} \sin ^{3} i\left(M_{\odot}\right)$ & 2.168 & \pm & 0.020 \\
$\sigma_{A}\left(\mathrm{~km} \mathrm{~s}^{-1}\right)$ & & 1.37 & \\
$\sigma_{B}\left(\mathrm{~km} \mathrm{~s}^{-1}\right)$ & & 1.28 & \\
\hline
\end{tabular}

${ }^{a}$ Time of periastron passage.

${ }^{b}$ The uncertainty in the RV of the template is not included. variation due to the eclipse, so that $(1-\alpha)$, e.g., is the fraction of the primary star light blocked during the primary eclipse.

When measuring spectral lines on a composite spectrum, the resulting equivalent widths differ from the intrinsic intensity of the lines by a factor $(1+Q)^{-1}$ for the primary spectrum and $Q(1+Q)^{-1}$ for the secondary one, with $Q$ the quotient of the continuum intensities. If one of the components is partially eclipsed, these factors change to $(1+\beta / \alpha Q)^{-1}$ and $\beta / \alpha Q(1+\beta / \alpha Q)^{-1}$, respectively.

Since the spectra $A$ and $B$ are calculated from observed spectra at phases out of eclipse, they reflect the relative luminosity of the stars. Thus, any observed spectrum may be described by

$f_{1} \cdot A\left(x-v_{a}\right)+f_{2} \cdot B\left(x-v_{b}\right)$

where $v_{a}$ and $v_{b}$ are the RVs of the components, and the scale factors are

$f_{1}=(1+Q) /(1+\beta / \alpha Q)$ 


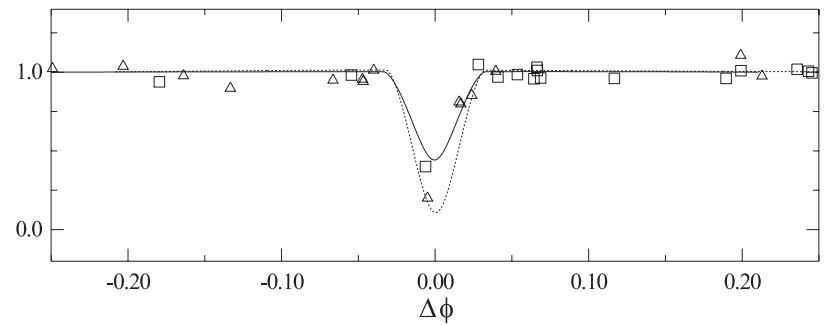

Fig. 4. Variation in the relative strength of the spectral lines of primary (triangles) and secondary (squares) components, as a function of the difference in the orbital phase with respect to the expected center of the primary and secondary eclipse, respectively. For comparison, theoretical curves for the line intensities of the primary (continuous lines) and the secondary (dashed line) components are plotted assuming an orbital inclination $i=87^{\circ}$.

and

$f_{2}=\beta / \alpha \cdot(1+Q) /(1+\beta / \alpha Q)$.

In order to obtain $\beta / \alpha$, we computed the difference $S(x)-f_{1}$. $A\left(x-v_{a}\right)-f_{2} \cdot B\left(x-v_{b}\right)$ for a grid of $\beta / \alpha$ for each observed spectrum, and found the optimal $\beta / \alpha$ factor that minimizes those residuals. Assuming that the light variations are mainly due to eclipses, we assumed $\alpha \equiv 1$ for spectra taken at phases when the primary component is closer to the observer, and $\beta \equiv 1$ for the opposite position. Figure 4 shows the resulting values of $\alpha$ and $\beta$.

This procedure for evaluating light variations from line intensities in an eclipsing binary is similar to the relative line photometry proposed by Hadrava (1997). The value of the light factor for the two spectra at conjunction is very uncertain because of the similarity of their spectral types and the small RV difference. However, it can be asserted that in both spectra most of the light of one component is absent. This analysis revealed that, besides the two spectra mentioned in Sect. 3.2, another three spectra are at phases of partial eclipse. This allows us to estimate the duration of the eclipses and, therefore, the sum of stellar radii for a given orbital inclination. In the next section this will be used to infer the absolute stellar parameters.

The spectroscopic detection of eclipses motivated us to search for photometric data to confirm our result. We found that Tycho (ESA 1997) photometry, in spite of its low precision, clearly shows light variations when the observations are phased with our spectroscopic ephemeris (see Fig. 5). More recently, while this paper was being written, Otero \& Dubovsky (2004) reported HD 143511 as a photometric binary using data from the ASAS-3 database (Pojmanski 2002). They publish a period of 5.5354 days and mention this binary as eccentric, although no further analysis of these photometric data has been done.

\section{Absolute parameters and age}

In this section we attempt to derive the absolute masses and sizes of the stellar components by analyzing all the spectroscopic information mentioned in the previous section. In Fig. 6 the sum of the radii is plotted against the total mass for the

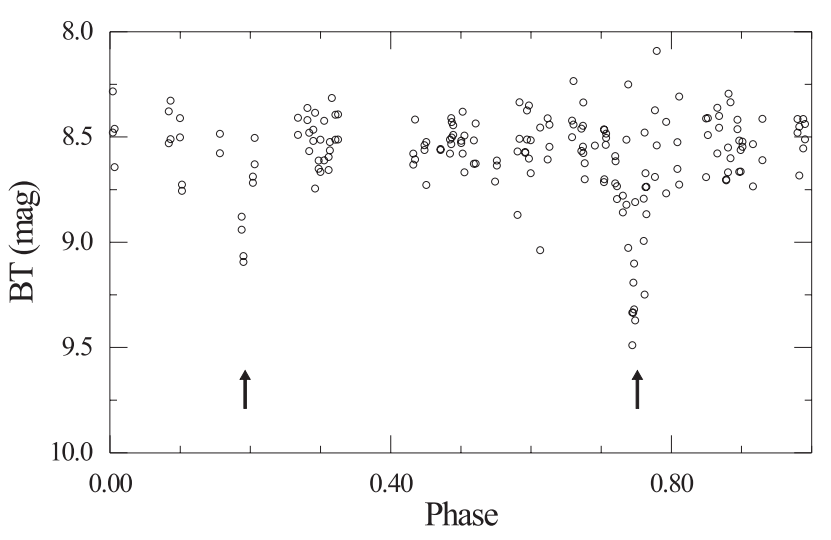

Fig. 5. Tycho Photometry of HD 143511 as a function of orbital phase computed from our spectroscopic ephemeris. Arrows mark the predicted phases of eclipse.

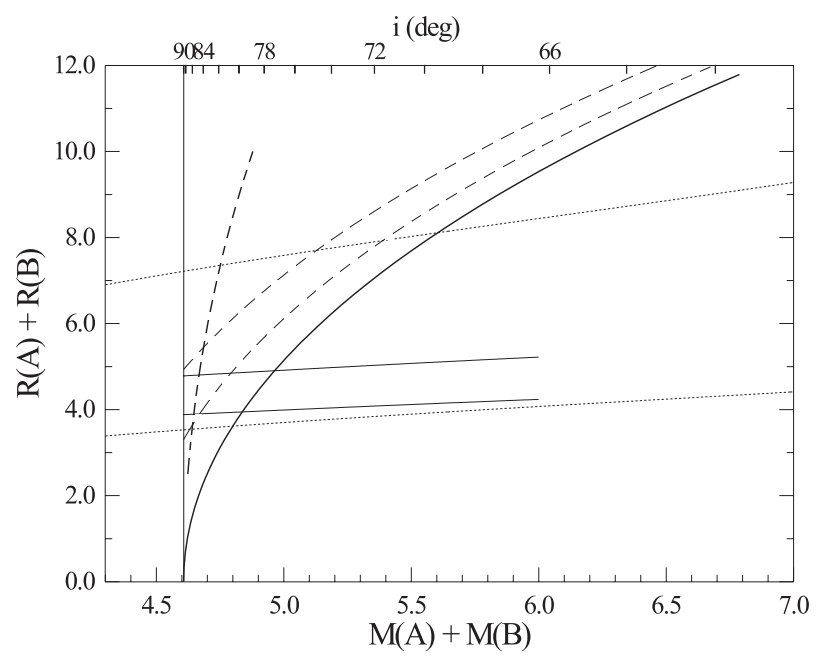

Fig. 6. Sum of radii vs. total-mass for the possible configurations of the binary HD 143511. See text.

possible configurations of the system. The dotted lines correspond to systems with both stars at ZAMS and to systems with the primary star at TAMS. These curves were calculated by interpolating in the theoretical stellar models by Girardi et al. (2000) for solar composition. The vertical line marks the lower limit for the total mass imposed by the orbital analysis, i.e., $\left(M_{A}+M_{B}\right) \sin ^{3} i$.

The thick continuous line corresponds to $R_{A}+R_{B}=r \cos i$, where $r$ is the separation of the stars at the conjunction phase. This line divides the eclipsing systems (up and left) from the non-eclipsing systems (right and down). We used the separation of the components $r$ calculated for the phase of the primary eclipse, although the difference with the one corresponding to the secondary eclipse is small (about 3\%), due to the small eccentricity and the orientation of the orbit. The phase extent of the eclipses gives information on the stellar radii. In our case, the primary eclipse is useful for this purpose, since the three spectra taken a short time before the end of this eclipse allow us to estimate its duration. From the relative luminosity curves of Fig. 4, we estimated the absolute value of the phase difference between the first contact and the center of the primary eclipse to be $\Delta \phi=0.030 \pm 0.006$. We then computed 
Table 3. Absolute parameters.

\begin{tabular}{llll}
\hline \hline$M_{A}\left(M_{\odot}\right)$ & 2.45 & \pm & 0.03 \\
$M_{B}\left(M_{\odot}\right)$ & 2.18 & \pm & 0.03 \\
$R_{A}\left(R_{\odot}\right)$ & 2.44 & \pm & 0.16 \\
$R_{B}\left(R_{\odot}\right)$ & 1.91 & \pm & 0.17 \\
\hline
\end{tabular}

the corresponding true anomaly difference $\Delta v$ from this value. Then, we calculated the sum of radii as a function of the orbital inclination $i$ using the following expression that is valid for the end of the primary eclipse:

$R_{A}+R_{B}=\frac{a\left(1-e^{2}\right)}{[1+e \sin (\omega-\Delta v)]}\left[\sin ^{2}(\Delta v)+\cos ^{2}(\Delta v) \cos ^{2} i\right]^{1 / 2}$.

The two thin dashed curves in Fig. 6 represent the sum of the radii for $\Delta \phi=0.024$ and 0.036 .

The weakness, or even non-detection, of the spectrum of the eclipsed component indicates a high orbital inclination. As mentioned in Sect. 4.3, most of the light of one of the stars is eclipsed for the two spectra centered in the conjunction phases. Synthetic light curve calculations show that, if at least half of the light of the star is blocked during the eclipse, then the orbital inclination is greater than $84^{\circ}$ (thick dashed line in Fig. 6), and the total mass of the system is confined to the range 4.61-4.68 $M_{\odot}$. This uncertainty is comparable to the spectroscopic error of the total mass $\left(0.042 M_{\odot}\right)$, so even without information from light curves, the stellar masses are defined within a $1-2 \%$ error. On the other hand, if we restrict the orbital inclination to $i=84^{\circ}-90^{\circ}$, then the eclipse duration $\Delta \phi$ gives the value $4.4 \pm 1.0 R_{\odot}$ for the sum of radii. The radius-ratio can be derived from the spectroscopic luminosity-ratio, if the temperatures are known. Using the temperature and luminosity-ratio obtained in Sect. 4, we get $R_{2} / R_{1}=0.79 \pm 0.08$.

Furthermore, the stellar radii can be estimated from the rotational velocity. For binary systems whose components rotate synchronously with the orbital motion, the rotational velocities provide the relative radii. Synchronization times are less than 1 Myr for this system according to theoretical models (Tassoul 1987, 1988), so it is reasonable to assume that the stellar rotation rate equals the orbital angular rate at periastron. Using a rotational period of 4.5581 days and an estimated orbital inclination $i=87 \pm 3 \mathrm{deg}$, we derived from the measured rotational velocities the following values for the stellar radii: $R_{A}=2.44 \pm 0.18 R_{\odot}$ and $R_{B}=1.89 \pm 0.27 R_{\odot}$. For high inclination configurations, the sum of the radii derived from rotational velocities, $\left(R_{A}+R_{B}\right) \sin i=4.32 \pm 0.45 R_{\odot}$, agrees with the value estimated above from the duration of the eclipse (thin continuous lines in Fig. 6).

Finally, we adopted the absolute parameters listed in Table 3, which are the results of combining the information from radial velocities, rotational velocities, eclipse duration, and ratio of spectral line intensities. Figure 7 shows the massradius diagram for the components of HD 143511 compared with stellar models of Girardi et al. (2000) for solar composition. The estimated radius of the primary component suggests an age of $\log \tau=8.5$. The stellar temperature corresponds to slightly more evolved models, approximately $\log \tau=8.6$.

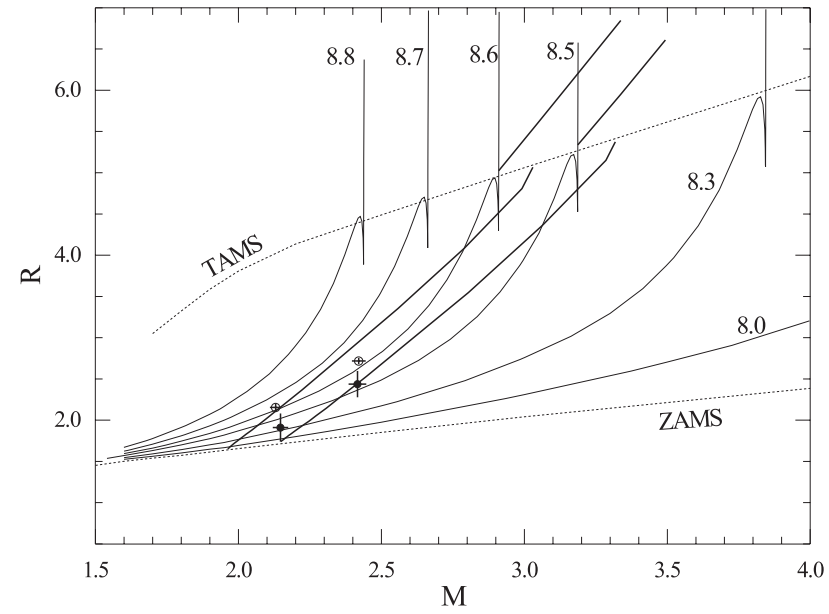

Fig. 7. Mass-radius diagram. The position of the stellar components of HD 143511 is compared with stellar models for solar composition. Filled circles with error bars correspond to the absolute parameters listed in Table 3; open circles are the parameters calculated using the photometric data of the ASAS-3 database (Pojmanski 2002). Isochrones are shown for $\log \tau$ 8.5, 8.6, and 8.7. Thick lines are isotherms for temperatures $9000 \mathrm{~K}$ and $9600 \mathrm{~K}$, corresponding to the range of temperatures adopted for both stars from the spectral types and the available photometry. The TAMS loop of isotherms has been omitted for clarity.

The photometric data existing in the ASAS-3 database (Pojmanski 2002) gives an opportunity to derive photometric radii to then be compared with our purely spectroscopic results. Even when we plan to obtain more complete and accurate light curves of this binary, the existing photometric data are good enough to improve our estimates of stellar radii. Using the Wilson \& Devinney program (Wilson \& Devinney 1971; Wilson 1990), we computed the orbital inclination and stellar radii to obtain the following values: $i=88.7 \pm 0.4 \mathrm{deg}$, $R_{A}=2.72 \pm 0.04 R_{\odot}$, and $R_{B}=2.15 \pm 0.05$, where the errors are standard errors computed by the Wilson \& Devinney program. These values for the radii are somewhat higher than those found from the spectroscopy (Fig. 7) and are very well-fitted by an isochrone of about $\log \tau=8.60-8.65$. The photometric radii are consistent, within the observational errors, with the radiusratio inferred from the spectroscopic luminosity-ratio and the sum of the radii calculated in Sect. 4.3 from the duration of the eclipse. On the other hand, the radii derived from the assumption of synchronic rotation are smaller. It is not clear whether the uncertainties of our $v \sin i$ are underestimated or if the synchronization hypothesis is not valid. Spectroscopic observations at higher resolution would be needed to get a conclusive result about the synchronization of the system.

\section{Discussion}

\subsection{The method}

We have presented a method to obtain reliable RVs of composite spectra and provided high quality spectra of the individual components. By shifting and combining the observed 
composite spectra, the lines of one of the components are removed, obtaining single-lined spectra that are used to measure RVs and to compute separate spectra for the two stellar components. Since all the observed spectra are combined for computing the primary and secondary spectra, the resulting signalto-noise is increased proportionally to the square root of the number of observed spectra. This fact allows us to perform a more detailed study of the spectroscopic features of the stellar components.

Even if the fundamentals of the Bagnuolo \& Gies (1991) tomography are essentially different, our algorithm for the computation of the spectra $A$ and $B$ has certain similarity to their tomography; compare Eqs. (1) and (2) with their Eqs. (3a) and (3b). Note that our spectra $A$ and $B$ correspond in the Bagnuolo \& Gies notation to $f_{1}$ and $r f_{2}$, respectively. However, in some aspects our computation of the component spectra differs from the tomographic method; in our case pixel shifts do not need to be integer values and averages may also differ from a pure arithmetic mean.

The present method was implemented entirely through IRAF tasks, which allows us to customize the computations for each particular case by modifying the task parameters, introducing weights, rejection algorithms, Fourier filtering, etc. The convergence rate during computation of the component spectra depends strongly on the orbital phase range covered by the observations and on the number of spectral lines present in the spectrum.

The method showed itself to be useful for measuring the RVs of blended spectra even for separations of only one third of the sum of the FWHM for the spectra lines, where classical methods fail to work. This is possible because it uses information on the spectral line width of individual components, which is also the case for the TODCOR technique of Zucker \& Mazeh (1994). For that reason, both methods give good RVs in high rotation binaries (see for example González \& Lapasset 2003). However, for computing the individual spectra of the components, it is necessary that the system can be resolved at quadrature, i.e., to have at least some observed spectra for which the $\mathrm{RV}$ of the components can be appropriately identified. This condition is the same for other similar methods. In the case of tomographic or disentangling methods (Bagnuolo \& Gies 1991; Simon \& Sturm 1994; Hadrava 1995), the difficult point is to provide starting values for the RV of the components. In the case of TODCOR, the problem is to define the spectral morphology, particularly the rotational width of the spectral lines on which the RV are strongly dependent for blended spectra.

Finally, we comment on the luminosity ratio of the components. As noted by other authors (Bagnuolo \& Gies 1991; Simon \& Sturm 1994) any method aimed at separating the components of a spectroscopic binary by combining observed spectra at different orbital phases is unable to determine the contribution of each star to the continuum level. This is a consequence of the invariance of the binary continuum with the orbital phase, since Doppler shifts are small in comparison with the wavelength scale of the variations in the stellar continuum. Consequently, if $A(x)$ and $B(x)$ are a solution to the problem of separating the two component spectra from a given sample of observed composite spectra, then $A(x)+f(x)$ and $B(x)-f(x)$ also provide a solution of the same problem for any function $f(x)$ with a slow dependence on $\lambda$. Note that it is unnecessary to know the luminosity-ratio before computing the spectra of the components, since they may be scaled afterwards. In fact, our adoption of $B^{0}=0$ leads to final spectra $A$ and $B$ with continuum level equal to 1 and 0 , respectively. To measure equivalent widths, the luminosity ratio has to be known from other sources, for example, from light curve analysis in eclipsing binaries, or by using the information of the absolute equivalent width expected for some spectral lines according to the spectral type of each component.

\subsection{The binary HD 143511}

In the second part of this paper we applied the method described above to the binary HD 143511. The RVs for both components were measured with errors of about $1.3 \mathrm{~km} \mathrm{~s}^{-1}$. The orbital analysis provided accurate $(1 \%)$ values for the minimum masses.

We detected the occurrence of eclipses spectroscopically thanks to the procedure of decoupling the stellar components in the spectrum. From different spectroscopic parameters we estimated the absolute stellar radii and the orbital inclination, obtaining in that manner absolute masses and dimensions. These results were then compared to the absolute radii derived using the photometric light curves recently published by Otero \& Dubovsky (2004). Both stellar components are main sequence stars.

Kinematically, HD 143511 is not a member of the cluster NGC 6025; its center-of-mass velocity differs by $38 \mathrm{~km} \mathrm{~s}^{-1}$ from the cluster RV $\left(+12 \mathrm{~km} \mathrm{~s}^{-1}\right.$, González \& Levato, in preparation). On the other hand, the absolute parameters indicate that the binary is considerably closer than the cluster and that it is older. Interpolating the absolute magnitude of each component in the Girardi et al. (2000) models according to the observed mass and radius, we calculated the absolute magnitude of the system. The resulting apparent distance modulus is $8.20 \pm 0.05$, or $8.02 \pm 0.09$ if the spectroscopic radii were used. On the other hand, the distance modulus of NGC 6025 is 9.95 (Meynet et al. 1993), which indicates that HD 143511 is closer than the cluster by a factor $0.4-0.5$ approximately.

The spectral analysis suggests a marginal chemical peculiarity, so we plan to perform a detailed chemical analysis in the near future. In addition, we plan to obtain photometric curves in order to derive absolute parameters for this system.

Acknowledgements. We appreciate the support from the Consejo Nacional de Investigaciones Científicas y Técnicas (CONICET) through a grant PIP 2147.

\section{References}

Bagnuolo, W. G., \& Gies, D. R. 1991, ApJ, 376, 266

ESA 1997, The Hipparcos and Tycho Catalogues (ESA SP-1200) (Noordwijk: ESA) 
Gies, D. R. 2004, in Spectroscopically and Spatially Resolving the Components of Close Binary Stars, ed. R. W. Hilditch, H. Hensberge, \& K. Pavlovski (San Francisco: ASP), ASP Conf. Ser., 318,61

Girardi, L., Bressan, A., Bertelli, G., \& Chiosi, C. 2000, A\&AS, 141, 371

González, J. F., \& Lapasset, E. 2003, A\&A, 404, 365

Hadrava, P. 1995, A\&AS, 114, 393

Hadrava, P. 1997, A\&AS, 122, 581

Hadrava, P. 2004, in Spectroscopically and Spatially Resolving the Components of Close Binary Stars, ed. R. W. Hilditch, H. Hensberge, \& K. Pavlovski (San Francisco: ASP), ASP Conf. Ser., 318,86

Holmgren, D. E. 2004, in Spectroscopically and Spatially Resolving the Components of Close Binary Stars, ed. R. W. Hilditch, H. Hensberge, \& K. Pavlovski (San Francisco: ASP), ASP Conf. Ser., 318,95

Houk, N., \& Cowley, A. P. 1975. Michigan Catalogue of twodimensional spectral types for the HD star, Ann Arbor: University of Michigan
Kilambi, G. C. 1975, PASP, 87, 975

Kukarkin, B. V., Kholopov, P. N., Artiukhina, N. M., et al. 1981, Nachrichtenblatt der Vereinigung der Sternfreunde, Moscow, Acad. of Sciences USSR Shternberg

Marchenko, S. V., Moffat, A. F. J., \& Eenens, P. J. R. 1998, PASP, 110, 1416

Meynet, G., Mermilliod, J.-C., \& Maeder, A. 1993, A\&AS, 98, 477

Napiwotzki, R., Schonberner, D., \& Wenske, V. 1993, A\&A, 168, 653

Otero, S. A., \& Dubovsky, P. A. 2004, IBVS, 5557, 1

Pojmanski, G. 2002, Acta Astron., 52, 397

Royer, F., Gerbaldi, M., Faraggiana, R., \& Gómez, A. E. 2002, A\&A, 381, 105

Simon, K. P., \& Sturm, E. 1994, A\&A, 281, 286

Strohmeier, W. 1967, IBVS, 178, 1

Tassoul, J. L. 1987, ApJ, 322, 856

Tassoul, J. L. 1988, ApJ, 324, L71

Wilson, R. E., \& Devinney, E. J. 1971, ApJ, 166, 605

Wilson, R. E. 1990, ApJ, 356, 613

Zucker, S., \& Mazeh, T. 1994, ApJ, 420, 806 